\title{
Correlation between obesity and pelvic organ prolapse in Korean women
}

\author{
Bo Hye Kim, MD', Soo Bin Lee, MD², Eun Duc Na, MD², Hyeon Chul Kim, MD² \\ Department of Obstetrics and Gynecology, ${ }^{1}$ Ymiz Woman Clinic, Anyang; ${ }^{2} \mathrm{CHA}$ Bundang Medical Center, CHA University, Seongnam, Korea
}

Objective

This study aimed to evaluate the correlation between obesity and pelvic organ prolapse (POP), both anatomically and symptomatically, in Korean women.

\section{Methods}

We retrospectively reviewed 476 women who visited the urogynecology clinic between January 2013 and December 2016. All the enrolled women were Korean. We sought to evaluate the relationship between obesity and POP, both anatomically and symptomatically, by using a validated tool. Anatomic assessment was performed by a standardized Pelvic Organ Prolapse Quantification (POP-Q) system and symptomatic assessment was performed by a Pelvic Floor Distress Inventory (PFDI)-20 questionnaire. Obesity measurement was performed by measuring body mass index (BMI).

Results

We enrolled 476 women in our study. There was no statistically significant correlation between BMI and POP-Q or PFDI-20 scores: $\mathrm{Ba}(P=0.633), \mathrm{Bp}(P=0.363), \mathrm{C}(P=0.277)$, Pelvic Organ Prolapse Distress Inventory- $6(P=0.286)$, Colorectal Anal Distress Inventory-8 $(P=0.960)$, Urinary Distress Inventory-6 $(P=0.355)$, and PFDI-20 $(P=0.355)$. In addition, there was no statistically significant correlation between BMI and POP-Q or PFDI-20 in patients with severe (greater than stage III) POP. We also separately analyzed the differences in the POP-Q points and PFDI-20 scores between the obese and non-obese groups. There was no statistically significant difference between the groups.

\section{Conclusion}

We evaluated the correlation between obesity and POP using a validated tool. The present study revealed no significant correlation between obesity and POP severity anatomically or symptomatically in Korean women. This contrasts the results of most studies of Western women. Further studies in Asian women are required in order to confirm our results.

Keywords: Pelvic organ prolapse; Obesity

\section{Introduction}

Pelvic organ prolapse (POP) is a descent of one or more aspects of the vagina and uterus, presumably due to defects in the pelvic organ support system. POP is a common condition in women, with prevalence rates ranging from $41 \%$ to $50 \%$ [1]. The lifetime risk of undergoing a single operation for POP or urinary incontinence is approximately $11 \%$ [2].

Many studies have been conducted in order to determine the risk factors for POP. Increasing parity and aging are established risk factors for POP; however, these factors are not modifiable $[3,4]$. Obesity is a modifiable risk factor; however, studies on the relationship between obesity and POP have
Received: 2019.05.02. Revised: 2020.05.08. Accepted: 2020.05.12. Corresponding author: Hyeon Chul Kim, MD

Department of Obstetrics and Gynecology, CHA Bundang Medical Center, CHA University, 59 Yatap-ro, Bundang-gu, Seongnam

13496, Korea

E-mail: philia@cha.ac.kr

https://orcid.org/0000-0002-4894-6140

Articles published in Obstet Gynecol Sci are open-access, distributed under the terms of the Creative Commons Attribution Non-Commercial License (http://creativecommons. org/licenses/by-nc/3.0/) which permits unrestricted non-commercial use, distribution, and reproduction in any medium, provided the original work is properly cited.

Copyright $\odot 2020$ Korean Society of Obstetrics and Gynecology 


\section{Obstetrics \& Gynecology Science}

Vol. 63, No. 6, 2020

shown inconsistent results. Many of these studies did not use a validated tool for the evaluation of POP. Obesity is associated with pelvic floor symptoms, as indicated by previous studies $[5,6]$.

Understanding the impact of obesity on POP is crucial as obesity is a modifiable risk factor whose prevalence is increasing, especially in women [7]. The diagnostic criteria for obesity differs between Western and Asian women. Moreover, studies in Asian women are scarce. The objective of this study was to evaluate the correlation between obesity and POP, both anatomically and symptomatically, in Korean women.

\section{Materials and methods}

We retrospectively reviewed the medical records of patients whose chief complaints were related to POP, and who visited the CHA Bundang Medical Center from January 2013 to December 2016. All of the enrolled women were Korean.

Each patient underwent a comprehensive physical examination, which included a standardized Pelvic Organ Prolapse Quantification (POP-Q) examination for the anatomical assessment of POP. A POP-Q examination was performed by a practitioner with the patient in the lithotomy position during the valsalva strain. At the initial visit, we recorded height, weight, and results of the POP-Q assessment: Ba as the most distal point of the upper anterior vaginal wall, $\mathrm{Bp}$ as the most distal point of the posterior vaginal wall, $\mathrm{C}$ as the most distal edge of the cervix or vaginal cuff, and total vaginal length (TVL) as the total length of the vagina. The POP degree was represented by the POP stage from 0 to IV based on the POPQ system [8]. Each patient was also asked to complete the PFDI-20 questionnaire at the initial visit. The PFDI-20 is a validated questionnaire used to assess the degree of pelvic floor symptom distress and is composed of 3 subscales: Pelvic Organ Prolapse Distress Inventory (POPDI)-6, Colorectal Anal Distress Inventory (CRADI)-8, and Urinary Distress Inventory (UDI)-6. Here, we used the PFDI-20 Korean translated version [9].

We evaluated obesity based on the patient's BMI. Generally, obesity is defined as a BMl $\geq 30 \mathrm{~kg} / \mathrm{m}^{2}$ and overweight is defined as a BMI $25-30 \mathrm{~kg} / \mathrm{m}^{2}$ according to the World Health Organization (WHO) guidelines [10]. However, here, we defined obesity as $\mathrm{BMI} \geq 25 \mathrm{~kg} / \mathrm{m}^{2}$ as defined previously for the Asia-Pacific population [10].
We analyzed the correlation between obesity and POP using continuous variables and dichotomous variables. We then separately evaluated the correlation between obesity and POP for patients with advanced POP (stages III and IV). We excluded women with incomplete records of height and weight, or POP stage $\leq 1$ at the initial visit. We also excluded women who were pregnant and/or underwent prior surgery due to POP.

We used 2 statistical methods. Pearson's correlation coefficient was used to analyze the correlation of BMI as a continuous variable with POP-Q points and PFDI-20 scores. An independent samples $t$-test was used to evaluate differences between the groups (obese/non-obese) for obesity as a dichotomous variable. The results were considered significant if the $P<0.05$. All statistical analysis was performed using SPSS software (version 24; SPSS Inc., Chicago, IL, USA).

\section{Results}

In total, 533 women were reviewed and the following

Table 1. Demographics and clinical characteristics of patients $(n=476)$

\begin{tabular}{lcc}
\hline Variables & No. (\%) & Mean \pm SD \\
\hline Mean age $(\mathrm{yr})$ & & $65.56 \pm 11.27$ \\
Mean BMI $\left(\mathrm{kg} / \mathrm{m}^{2}\right)$ & $24.35 \pm 3.08$ \\
$<25$ (non-obese) & $188(39.5)$ & \\
$\geq 25$ (obese) & $167(88.8)$ & \\
$\geq 25$ and $<30$ & $21(11.1)$ & \\
$\geq 30$ & & $3.19 \pm 1.50$ \\
Mean parity & $436(91.6)$ & \\
Post menopausal status & $23(4.8)$ & \\
Prior pessary use & $43(9.3)$ & \\
Prior hysterectomy & $4(0.8)$ & \\
Hormonal therapy & \\
POP-Q stage & $139(29.2)$ \\
Stage II & $293(61.6)$ \\
Stage III & $44(9.2)$ \\
Stage IV & \\
Race & $476(100.0)$ \\
Asian &
\end{tabular}

SD, standard deviation; BMl, body mass index; POP-Q, Pelvic Organ Prolapse Quantification. 


\section{Obstetrics \& Gynecology Science}

Bo Hye Kim, et al. Obesity and pelvic organ prolapse

women were excluded: 27 patients with incomplete records of height and weight, 3 with POP stage $\leq 1,15$ who underwent hysterectomy due to POP, 8 who underwent prior prolapse surgery, and 4 who were pregnant at the initial visit. Ultimately, 476 women who met the inclusion criteria were enrolled and completed the POP-Q exam. Among the 476 enrolled patients, 370 (77.7\%) patients completed the PFDI20 questionnaire, $223(80.9 \%)$ patients were in the nonobese group, and 147 (78.2\%) patients were in the obese group. The study subjects had a mean age of $65.56 \pm 11.27$ years (91.6\% were postmenopausal), a mean BMI of $24.35 \pm 3.08 \mathrm{~kg} / \mathrm{m}^{2}$, and a mean parity of $3.19 \pm 1.50$ (Table 1). The largest group from the POP-Q belonged to stage III and the distribution of the POP-Q points are described in Table 2 . All subjects were divided into two groups, an obese group $(n=188,39.5 \%)$ and a non-obese group ( $n=288,60.5 \%)$, based on the BMI cut-off value of $25 \mathrm{~kg} / \mathrm{m}^{2}$. There were no statistically significant differences in age, parity, and/or prior hysterectomy between the two groups.
To evaluate the correlation between obesity and POP anatomically, we calculated Pearson's correlation coefficient for $\mathrm{BMI}$ and POP-Q points ( $\mathrm{Ba}, \mathrm{Bp}$, and $\mathrm{C})$. There were no statistically significant correlations between $\mathrm{BMI}$ or each POPQ point (Table 3, Fig. 1). In the same manner, to evaluate the correlation with obesity and POP symptomatically, we calculated Pearson's correlation coefficient for BMI and PFDI-20 and its subscales (POPDI-6, CRADI-8, and UDI-6). There was no statistically significant correlations between BMI and PFDI20 (Table 3, Fig. 2). In the same manner, we also evaluated the correlation between obesity and POP for patients with severe POP (stage $\geq I I I)$. There was no statistically significant correlations between BMI, POP-Q, or PFDI-20 (Table 4) in patients with severe POP. All enrolled patients were dichotomously classified as obese or non-obese. We then separately analyzed the difference between the anatomic and symptomatic correlations between the two groups. No statistically significant anatomical or symptomatic difference was found between the obese and the non-obese groups (Table 5).

Table 2. Distribution of pelvic organ prolapse (POP) stage $(n=476)$

\begin{tabular}{lrrrrr}
\hline POP-Q points & Stage 0 & Stage I & Stage II & Stage III & Stage IV \\
\hline $\mathrm{Ba}$ & $5(1.0)$ & $49(10.3)$ & $145(30.5)$ & $240(50.4)$ & $37(7.8)$ \\
$\mathrm{Bp}$ & $11(2.3)$ & $100(21.0)$ & $259(54.4)$ & $69(14.5)$ & $37(7.8)$ \\
$\mathrm{C}$ & $17(3.6)$ & $188(39.5)$ & $89(18.7)$ & $141(29.6)$ & $41(8.6)$ \\
\hline
\end{tabular}

Data are presented as number (\%).

POP-Q, Pelvic Organ Prolapse Quantification.

Table 3. Correlation between body mass index (BMI) with Pelvic Organ Prolapse Quantification (POP-Q) points and Pelvic Floor Distress Inventory (PFDI)-20

\begin{tabular}{lcc}
\hline Variables & $\begin{array}{c}\text { Pearson's correlation } \\
\text { coefficient }\end{array}$ & P-value \\
\hline POP-Q points & 0.022 & 0.633 \\
Ba & -0.042 & 0.363 \\
Bp & -0.050 & 0.277 \\
C & & \\
PFDI-20 & 0.056 & 0.286 \\
POPDI-6 & 0.003 & 0.960 \\
CRADI-8 & 0.051 & 0.340 \\
UDI-6 & 0.048 & 0.355 \\
PFDI-20 & & \\
\hline
\end{tabular}

POPDI, Pelvic Organ Prolapse Distress Inventory; CRADI, Colorectal Anal Distress Inventory; UDI, Urinary Distress Inventory.

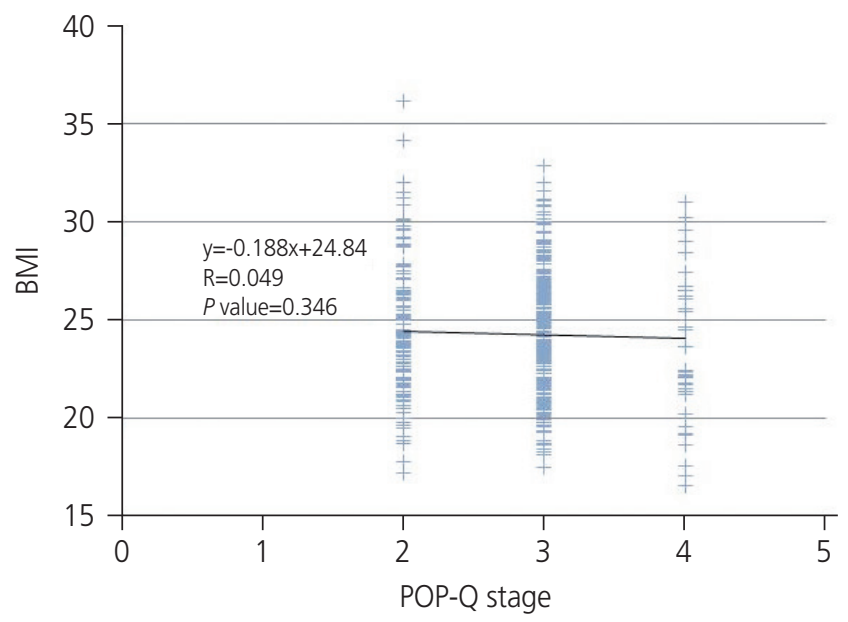

Fig. 1. Correlation between body mass index (BMI) and Pelvic Organ Prolapse Quantification (POP-Q) stage. BMI was not significantly correlated with POP-Q stage $(R=0.049, P=0.346)$. 


\section{Obstetrics \& Gynecology Science}

Vol. 63, No. 6, 2020

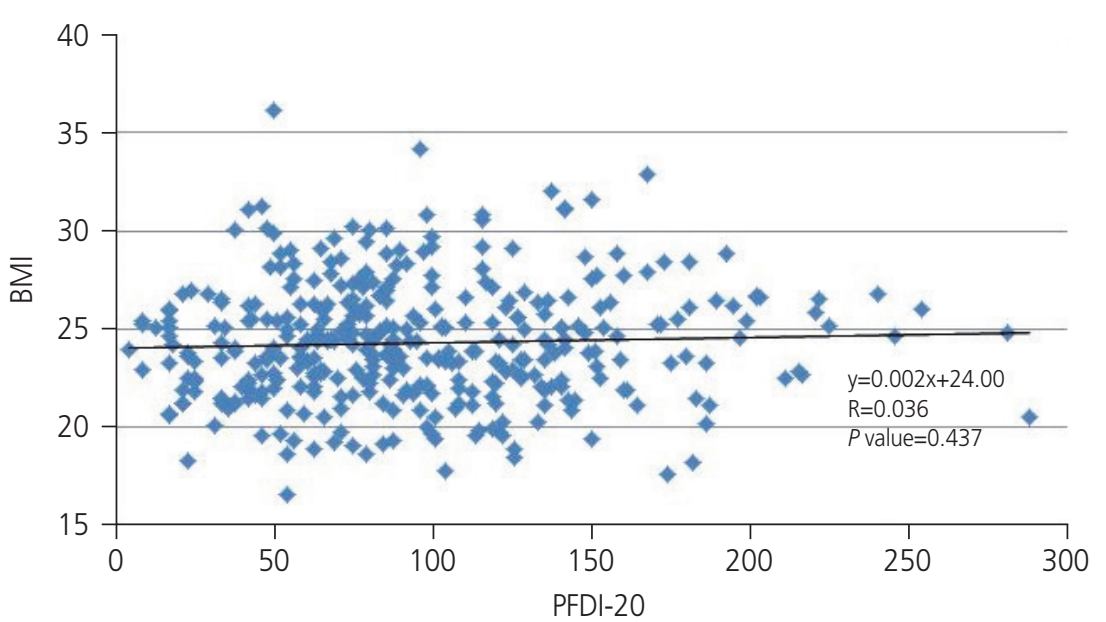

Fig. 2. Correlation between body mass index (BMI) and Pelvic Floor Distress Inventory (PFDI)20. BMI was not significantly correlated with PFDI-20 ( $R=0.036, P=0.437)$.
Table 4. Correlation between body mass index (BMI) with Pelvic Organ Prolapse Quantification (POP-Q) points and Pelvic Floor Distress Inventory (PFDI)-20 for $\geq$ stage 3 ( $n=337$ )

\begin{tabular}{lcc}
\hline Variables & $\begin{array}{c}\text { Pearson's correlation } \\
\text { coefficient }\end{array}$ & P-value \\
\hline POP-Q point & 0.026 & 0.571 \\
$\mathrm{Ba}$ & -0.040 & 0.381 \\
$\mathrm{Bp}$ & -0.055 & 0.227 \\
$\mathrm{C}$ & & \\
PFDI-20 & 0.048 & 0.359 \\
POPDI-6 & 0.005 & 0.924 \\
CRADI-8 & 0.049 & 0.357 \\
UDI-6 & 0.045 & 0.391 \\
PFDI-20 & & 0.06 \\
\hline
\end{tabular}

POPDI, Pelvic Organ Prolapse Distress Inventory; CRADI, Colorectal Anal Distress Inventory; UDI, Urinary Distress Inventory.

\section{Discussion}

Obesity has been consistently identified as a risk factor for pelvic floor disorder. The most probable mechanism of POP development in obese women is the increased intraabdominal pressure that causes weakening of the pelvic floor muscles and fascia [11]. However, previous studies that evaluated the relationship between obesity and POP illustrated inconsistent results. The prevalence of obesity has been increasing globally, and a better understanding of how obesity influences POP is crucial as unlike age and parity, obesity is a modifiable factor.
Table 5. Difference in Pelvic Organ Prolapse Quantification (POPQ) points and Pelvic Floor Distress Inventory (PFDI)-20 between obese/non-obese groups

\begin{tabular}{lccc}
\hline Variables & Obese group & $\begin{array}{c}\text { Non-obese } \\
\text { group }\end{array}$ & P-value \\
\hline POP-Q points & $1.9 \pm 2.3$ & $1.5 \pm 2.3$ & 0.052 \\
Ba & $0.3 \pm 2.2$ & $0.3 \pm 2.5$ & 0.874 \\
Bp & $-0.1 \pm 3.8$ & $-0.3 \pm 3.6$ & 0.574 \\
C & & & \\
PFDI-20 & $47.5 \pm 23.9$ & $43.2 \pm 22.4$ & 0.083 \\
POPDI-6 & $19.9 \pm 18.8$ & $19.0 \pm 18.2$ & 0.537 \\
CRADI-8 & $33.6 \pm 24.1$ & $30.3 \pm 22.0$ & 0.191 \\
UDI-6 & $99.1 \pm 53.7$ & $90.8 \pm 49.7$ & 0.131 \\
PFDI-20 & & & \\
\hline
\end{tabular}

Data are presented as mean \pm standard deviation.

POPDI, Pelvic Organ Prolapse Distress Inventory; CRADI, Colorectal Anal Distress Inventory; UDI, Urinary Distress Inventory.

In our study, obesity was not shown to be significantly related to POP anatomically or symptomatically. In contrast to our study, Hendrix et al. reported that there was a significant association between $\mathrm{BMI}$ and POP in overweight (BMI $\left.25-30 \mathrm{~kg} / \mathrm{m}^{2}\right)$ and obese women $\left(\mathrm{BMl} \geq 30 \mathrm{~kg} / \mathrm{m}^{2}\right)$ via data from the Women's Health Initiative study [12]. In that study, POP was assessed by visual inspection during the valsalva maneuver in the supine position, not by a standardized POP$Q$ exam. Shalom et al. [13] evaluated the correlation between obesity and POP by BMI and a standardized POP-Q exam. Consistent with our study, they reported that no significant correlation was established between BMI and POP severity. 


\section{Obstetrics \& Gynecology Science}

Bo Hye Kim, et al. Obesity and pelvic organ prolapse

Giri et al. published a systematic review and meta-analysis of the association between obesity and POP [14], and dichotomously assessed the risk ratio between obese and nonobese women (as defined by WHO). In contrast to our study, obesity was significantly associated with POP in their study. Compared with normal weight women (BMl $<25 \mathrm{~kg} / \mathrm{m}^{2}$ ), overweight women (BMI $25-30 \mathrm{~kg} / \mathrm{m}^{2}$ ) and obese women $\left(\mathrm{BMI} \geq 30 \mathrm{~kg} / \mathrm{m}^{2}\right)$ had risk ratios of at least 1.36 (95\% confidence interval [Cl], 1.20-1.53) and 1.47 (95\% Cl, 1.35-1.59), respectively. The authors did not include studies that used a continuous variable analysis. Vergeldt et al. [15] performed a systematic review of risk factors for POP and recurrence. In that study, a higher BMI as a dichotomous variable was a significant risk factor; however, $\mathrm{BMI}$ as a continuous variable had no association with POP. We performed dichotomous variable and continuous variable analyses to confirm the discrepancy between the two analyses. However, there were no significant correlations between either analyses.

Symptom distress due to POP is clinically significant and an investigation of the correlation between obesity and POP symptoms via a validated tool is also important. However, the correlation between obesity and POP symptom distress has been inconsistently reported in previous studies. We evaluated the correlation by a validated tool (PFDI-20 and its subscales), and no significant correlation was noted in our study. Uustal Fornell et al. [16] reported that being overweight or obese were strongly related to urinary and fecal incontinence, but not to POP symptoms. Bradley et al. [17] did not find a positive correlation between obesity and prolapse symptoms. This is consistent with our results. We also attempted to evaluate the correlation in patients with more severe prolapse. However, there was no significant correlation between obesity and POP anatomically or symptomatically in patients with severe POP (greater than stage III). Consistent with our findings, Shalom et al. [13] and Washington et al. [18] found that there was no significant correlation between $\mathrm{BMI}$ and prolapse severity.

With respect to obesity, there are differences between Korean women and Western women. According to the WHO guidelines, the cut-off value of obesity is defined as a BMI $\geq 30 \mathrm{~kg} / \mathrm{m}^{2}$, which was the cut-off value used in previous studies. However, the WHO obesity cut-off value for obesity for Asians is $25 \mathrm{~kg} / \mathrm{m}^{2}$; therefore, here, we defined obesity as a $\mathrm{BMI} \geq 25 \mathrm{~kg} / \mathrm{m}^{2}$. In our study, only $4.4 \%(21 / 476)$ of the patients had a BMI $\geq 30 \mathrm{~kg} / \mathrm{m}^{2}$. In contrast, $33.4 \%$ of US women had a BMI $\geq 30 \mathrm{~kg} / \mathrm{m}^{2}$ [19]. Therefore, with respect to the correlation between obesity and POP, a study for Korean women may have clinical significance.

However, studies for Korean women are rare and have demonstrated conflicting results. Kim et al. evaluated the risk factors for pelvic organ prolapse, and found that BMI was not significantly correlated with POP stage $(P=0.271)$ [20]. Seo and Kim [21] evaluated pelvic organ support and the prevalence of POP via the POP-Q exam for Korean women. In their study, BMI showed a significant correlation with increasing POP-Q stage $(P<0.001)$. Furthermore, the correlation of obesity and POP was not the primary aim in the above studies. To our knowledge, this is the first study that comprehensively evaluated the relationship between obesity and POP in Korean women. Although controversy exists, most of the studies for Western women showed a significant correlation between obesity and POP. Though diagnostic criteria for obesity is different between Western and Asian women, the present study showed that there was no significant correlation between obesity and POP severity anatomically or symptomatically in Korean women. The cause of different results between Western and Asian women is not clear. Further large, well-designed studies in Asian women may explain the discrepancy in these results.

The strength of this study is that we evaluated the correlation between obesity and POP anatomically as well as symptomatically. Furthermore, we also analyzed the data by dichotomous and continuous variables. Another strength is that we used a standardized tool for the anatomic and symptomatic evaluation of POP (POP-Q was used for anatomic evaluation, and PFDI-20 for symptomatic evaluation). There are some limitations of this study. First, this was a retrospective and cross-sectional study. Second, the enrolled women were restricted to patients with POP, therefore potentially limiting the generalizability of our results. Third, all patients underwent the POP-Q exam, but not all patients completed the PFDI-20, which may result in bias in the interpretation.

In conclusion, obesity was not significantly correlated with POP severity, anatomically or symptomatically, in Korean women. As noted earlier, diagnostic criteria for obesity is different between Western and Asian women and studies for Asian women are very scarce. Thus, further studies in Asian women are essential in order to confirm our findings. 


\section{Obstetrics \& Gynecology Science}

Vol. 63, No. 6, 2020

\section{Conflict of interest}

No potential conflict of interest relevant to this article was reported.

\section{Ethical approval}

This study was approved by the Institutional Review Board (IRB) of CHA Bundang Medical Center (IRB No. 2018-12026) and performed in accordance with the principles of the Declaration of Helsinki.

\section{Patient consent}

Informed consent was waived because of the retrospective study design.

\section{References}

1. Barber MD, Maher C. Epidemiology and outcome assessment of pelvic organ prolapse. Int Urogynecol J Pelvic Floor Dysfunct 2013;24:1783-90.

2. Olsen AL, Smith VJ, Bergstrom JO, Colling JC, Clark AL. Epidemiology of surgically managed pelvic organ prolapse and urinary incontinence. Obstet Gynecol 1997:89:501-6.

3. Nygaard I, Barber MD, Burgio KL, Kenton K, Meikle S, Schaffer J, et al. Prevalence of symptomatic pelvic floor disorders in US women. JAMA 2008;300:1311-6.

4. Mant J, Painter R, Vessey M. Epidemiology of genital prolapse: observations from the Oxford Family Planning Association Study. Br J Obstet Gynaecol 1997;104:57985.

5. Richter HE, Burgio KL, Clements RH, Goode PS, Redden DT, Varner RE. Urinary and anal incontinence in morbidly obese women considering weight loss surgery. Obstet Gynecol 2005;106:1272-7.

6. Erekson EA, Sung VW, Myers DL. Effect of body mass index on the risk of anal incontinence and defecatory dysfunction in women. Am J Obstet Gynecol 2008;198:596.e1-4.

7. Leeners B, Geary N, Tobler PN, Asarian L. Ovarian hor- mones and obesity. Hum Reprod Update 2017;23:30021.

8. Bump RC, Mattiasson A, Bø K, Brubaker LP, DeLancey JO, Klarskov $P$, et al. The standardization of terminology of female pelvic organ prolapse and pelvic floor dysfunction. Am J Obstet Gynecol 1996;175:10-7.

9. Yoo EH, Jeon MJ, Ahn KH, Bai SW. Translation and linguistic validation of Korean version of short form of pelvic floor distress inventory-20, pelvic floor impact questionnaire-7. Obstet Gynecol Sci 2013;56:330-2.

10. Yoon JL, Cho JJ, Park KM, Noh HM, Park YS. Diagnostic performance of body mass index using the Western Pacific Regional Office of World Health Organization reference standards for body fat percentage. J Korean Med Sci 2015;30:162-6.

11. Hunskaar S. A systematic review of overweight and obesity as risk factors and targets for clinical intervention for urinary incontinence in women. Neurourol Urodyn 2008;27:749-57.

12. Hendrix SL, Clark A, Nygaard I, Aragaki A, Barnabei V, McTiernan A. Pelvic organ prolapse in the Women's Health Initiative: gravity and gravidity. Am J Obstet Gynecol 2002;186:1160-6.

13. Shalom DF, Lin SN, St Louis S, Winkler HA. Effect of age, body mass index, and parity on Pelvic Organ Prolapse Quantification system measurements in women with symptomatic pelvic organ prolapse. J Obstet Gynaecol Res 2012;38:415-9.

14. Giri A, Hartmann KE, Hellwege JN, Velez Edwards DR, Edwards TL. Obesity and pelvic organ prolapse: a systematic review and meta-analysis of observational studies. Am J Obstet Gynecol 2017;217:11-26.e3.

15. Vergeldt TF, Weemhoff $M$, IntHout J, Kluivers KB. Risk factors for pelvic organ prolapse and its recurrence: a systematic review. Int Urogynecol J Pelvic Floor Dysfunct 2015;26:1559-73.

16. Uustal Fornell E, Wingren G, Kjølhede P. Factors associated with pelvic floor dysfunction with emphasis on urinary and fecal incontinence and genital prolapse: an epidemiological study. Acta Obstet Gynecol Scand 2004;83:383-9.

17. Bradley CS, Zimmerman MB, Wang Q, Nygaard IE; Women's Health Initiative. Vaginal descent and pelvic floor symptoms in postmenopausal women: a longitudinal study. Obstet Gynecol 2008;111:1148-53. 


\section{Obstetrics \& Gynecology Science}

Bo Hye Kim, et al. Obesity and pelvic organ prolapse

18. Washington BB, Erekson EA, Kassis NC, Myers DL. The association between obesity and stage II or greater prolapse. Am J Obstet Gynecol 2010;202:503.e1-4.

19. Foley JT, Lloyd M, Temple VA. Body mass index trends among adult U.S. Special Olympians, 2005-2010. Adapt Phys Activ Q 2013;30:373-86.
20. Kim CM, Jeon MJ, Chung DJ, Kim SK, Kim JW, Bai SW. Risk factors for pelvic organ prolapse. Int J Gynaecol Obstet 2007;98:248-51.

21. Seo JT, Kim JM. Pelvic organ support and prevalence by Pelvic Organ Prolapse-Quantification (POP-Q) in Korean women. J Urol 2006;175:1769-72. 